\title{
CFD Simulation of Biosurfactant Flooding into a Micromodel for Enhancing the Oil Recovery
}

\author{
Arezou Jafari, Seyed Ehsan Feghhi Pour, and Reza Gharibshahi
}

\begin{abstract}
In this research, the effect of biosurfactant flooding on the oil recovery factor using a CFD method is investigated. The porous medium is a $2 \mathrm{D}$ micromodel, and COMSOL Multiphysics 4.3 software is used to create the geometry and solving the governing equations on the system. Because of two different fluids exist into the porous medium; volume of fluid multiphase model was used for studying the fluid flow in the micromodel. Comparison of numerical results with the experimental data was done and a good agreement was observed between them. In addition, numerical data show that addition of the biosurfactant to the injected fluid causes an enhancement in the oil recovery factor and decreases the fingering effect. Also by biosurfactant flooding the breakthrough time increases, the surface tension between injected fluid and oil reduces and more surface area of the micromodel will be in contact with the injected fluid. So the oil recovery factor improves. Of course it should be mentioned that the shear stress acting on the biosurfactant is very harmful and it reduces its performance.
\end{abstract}

Index Terms-Biosurfactant, CFD, micromodel, oil recovery factor.

\section{INTRODUCTION}

With the development of industry and the lack of available energy resources, supply of new energy sources is vital. In the meantime the underground resources such as oil reservoirs are the best candidate to compensate for the shortage of energy [1]. But by natural forces in the reservoirs a little amount of oil can produce and to access the remaining oil in place another methods must be used. This methods are called enhanced oil recovery (EOR) techniques [2].

One of the best EOR methods is using microorganisms and their products to recover the oil in reservoirs that is known as microbial enhanced oil recovery (MEOR) [3]. MEOR operations have many advantages such as easy application [4], easy to handle, economic attraction and less expensive set up [5], and also low energy input requirement [6]. MEOR methods have great potential in carbonate reservoirs [4], and specially by reducing the surface tension [7] can increase the oil recovery factor [8].

It should be mentioned that this method is divided into two strategy in-situ and ex-situ [9], and the mechanism of microorganisms to improve the oil recovery factor is very diverse. In other words, bacteria as one of the important microorganisms by producing different metabolites such as

Manuscript received April 15, 2016; revised August 17, 2016. This work was supported by Laboratory Facilities of Tarbiat Modares University and Department of chemical engineering and sponsored by Iran's National Elites Foundation.

Authors are with Chemical Engineering Department, Tarbiat Modares University, Tehran, Iran (e-mail: ajafari@modares.ac.ir). biogas, bioacid, biosolvent, biopolymer, biosurfactant, biomass and etc. enhances the oil recovery [10], [11]. So according to the mentioned advantages for this process, a careful study of this method and removing obstacles facing can help to use this method in the field scale.

CFD technique is a powerful method to study the complex phenomena and analyzing the fluid flow in a system [12], [13]. In comparison with experimental methods, this technique reduces the time and cost, simulates the complex and difficult conditions easily and obtains more information about the problem [14]. But till now this technique has not been used to simulate MEOR operations.

Therefore, the aim of this work is simulation of the biosurfactant flooding using Bacillus mojavensis (JF-2, ATCC 39307) into a micromodel as a porous medium for the first time and studying the effect of this process on the oil recovery factor. It is worthy to note that micromodels are new apparatus that helps researchers to visualize the fluid flow into the porous medium and have an accurate study [15].

\section{NUMERICAL IMPLEMENTATION}

\section{A. Geometry Creation}

COMSOL Multiphysics 4.3 software was used to create the geometry of micromodel as the porous medium. The schematic of simulated micromodel and its dimensions are shown in Fig. 1 that is similar to the experimental study [16]

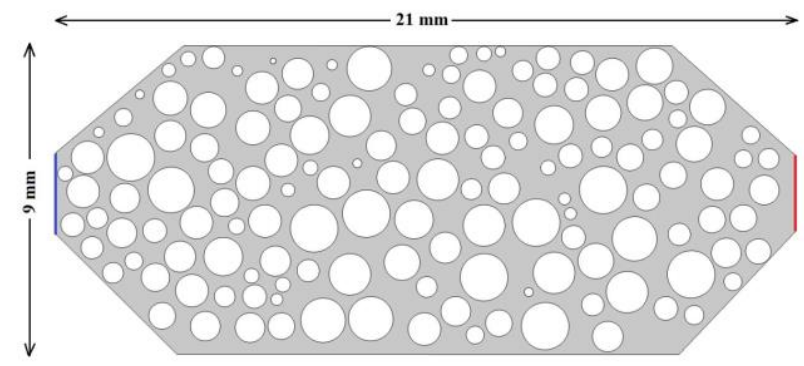

Fig. 1. The geometry of the micromodel.

To create the geometry 140 circles with different diameter (from 0.6 to $1.4 \mathrm{~mm}$ ) distributed in the main domain of the porous medium. In other word, 35 percent of circles have diameter $0.6 \mathrm{~mm}, 35$ percent of circles have diameter $0.8 \mathrm{~mm}$ and 30 percent of circles have diameter between 1.0 to 1.4 $\mathrm{mm}$. After subtracting the surface area of circles from the main domain, a porous medium with $52 \%$ porosity produces. It should be noted that the generated geometry is $2 \mathrm{D}$. In other words, regarding negligible measure of the model's thickness (50 micrometers) in relation to its other dimensions, its thickness was neglected and a two dimensional model was 
considered. The inlet and outlet ports are the left and right side of the micromodel, respectively.

\section{B. Mesh Generation}

Triangular unstructured mesh type was used for meshing the created geometry. The meshed geometry used for simulations has been presented in Fig. 2. In this figure for better visualization of the generated mesh, two zones of the porous medium are magnified.

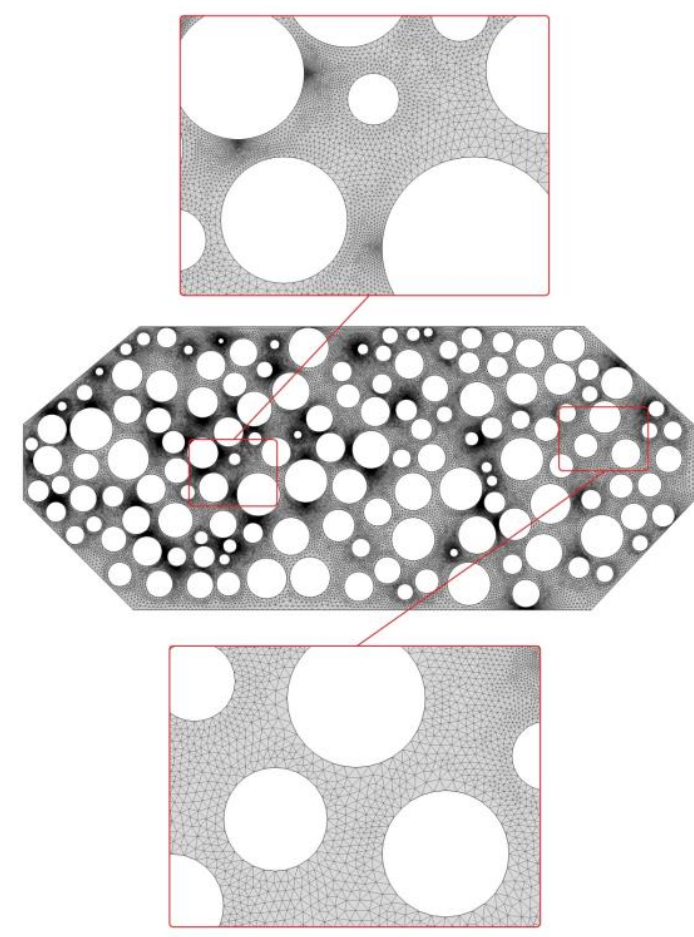

Fig. 2. The grid used in this study and magnified zones of the meshed geometry for better visualization.

It is obvious that to have a good simulation results, the numerical data must be grid independent. For this purpose, three grids with different mesh sizes were produced, and their parameters are tabulated in Table I.

TABLE I: CHARACTERIZATIONS OF GRIDS

\begin{tabular}{ccccc}
\hline \hline \multirow{2}{*}{ No. } & \multicolumn{3}{c}{ Value of ranges } & \multirow{2}{*}{ No. of Cell } \\
\cline { 2 - 4 } & Initial value & Growth rate & Final value & \\
\hline 1 & 0.0035 & 1.08 & 0.2 & $1.1 \times 10^{5}$ \\
2 & 0.0035 & 1.14 & 0.2 & $2.0 \times 10^{5}$ \\
3 & 0.0035 & 1.20 & 0.2 & $3.1 \times 10^{5}$ \\
\hline \hline
\end{tabular}

\section{Boundary Conditions and Assumptions}

The boundary conditions that applied on the system were velocity inlet and pressure outlet for the inlet and outlet port, respectively. The other walls of the micromodel (the main domain and circles) have been considered as wetted wall.

In this study, it is assumed that the bioclogging effect is negligible. In addition, the contact angle of oil/water was considered as $19^{\circ}$. Also for the initial state, the micromodel was saturated with crude oil with density $827 \mathrm{Kg} / \mathrm{m}^{3}$ and viscosity $0.087 \mathrm{~Pa}$.

To obtain the initial oil recovery, water flooding with constant initial velocity $10^{-4} \mathrm{~m} / \mathrm{s}$ was performed. Viscosity, density and the surface tension for water were assumed as
0.00103 Pa.s, $998 \mathrm{Kg} / \mathrm{m}^{3}$ and 48 dyne/cm, respectively. After that, for the second oil recovery, ex-situ biosurfactant flooding was done with the same inlet velocity, and the surface tension was considered as $0.4 \mathrm{dyne} / \mathrm{cm}$ according to the measured value in the research of Armstrong et al. [16]. Also, for inlet velocity $10^{-2} \mathrm{~m} / \mathrm{s}$ and surface tensions 0.2 and 0.3 dyne/cm calculations were done.

\section{Mathematical Formula}

In this research, a multiphase volume of fluid CFD model was used for investigation of fluid flow in the porous medium. Tracking the intersection among phases is performed by solving the continuity equation for volume fraction of one of the phases. For the $i^{\text {th }}$ phase, this equation is as follow [17]:

$$
\frac{1}{\rho_{i}}\left[\frac{\partial}{\partial t}\left(\alpha_{i} \rho_{i}\right)+\nabla \cdot\left(\alpha_{i} \rho_{i} \vec{v}_{i}\right)=S_{a i}+\sum_{j=1}^{n}\left(\dot{m}_{j i}-\dot{m}_{i j}\right)\right.
$$

Which $\mathrm{m}_{\mathrm{ji}}$ is the mass transfer from $\mathrm{i}^{\text {th }}$ phase to $\mathrm{j}^{\text {th }}$ phase. Regarding number of phases, volume fraction equation is solved for a smaller phase and the remained volume fraction is calculated according to the following restriction:

$$
\sum_{i=1}^{n} \alpha_{i}=1.0
$$

The momentum equation is solved all along the domain and the obtained velocity field lies in common possession of all the phases. The following momentum equation is dependent of volume fractions of all phases and fluid properties including viscosity and density:

$$
\frac{\partial}{\partial t}(\rho \vec{v})+\nabla \cdot(\rho \vec{v} \vec{v})=-\nabla p+\nabla \cdot\left[\mu\left(\nabla \vec{v}+\vec{v}^{T}\right)\right]+\rho_{i} \vec{g}+\vec{F}
$$

The volume of fluid (VOF) model considers the interfacial tension of each couple of phases. Also, this model can be completed by receiving the contact angle value.

COMSOL 4.3 software was used for simulation of the problem. For calculations of multiphase VOF model, phase-coupled simple method (PC-SIMPLE) with pressure based solver was used. PC-SIMPLE method was used to couple pressure-velocity. Velocities were solved as coupled with phases in discretization method. In this method, momentum and continuity equations are solved together based on pressure; therefore, rate of result convergence in comparison with discrete method which solves these equations repeatedly, improves. Convergence criterion has been set to $10^{-4}$ which is sufficient for most common problems. Unsteady simulations with time step 0.01 second was used, and under-relaxation factors which show the effect of each equation on final result have been presented in Table II.

TABLE II: UNDER-RELAXATION FACTORS

\begin{tabular}{cc}
\hline \multicolumn{2}{c}{ TABLE II: UNDER-RELAXATION FACTORS } \\
\hline Variable & Under-relaxation factor \\
\hline pressure & 0.6 \\
density & 1 \\
Body forces & 1 \\
momentum & 0.7 \\
\hline \hline
\end{tabular}

For implementing the process of simulation, a SuperMicro® computer with 24 processors and 16 GB ram 
was used, and in order to evaluate the performance of model the residual oil saturation $\left(\mathrm{S}_{\mathrm{or}}\right)$ is calculated by the following equation:

$$
S_{o r}(\%)=\frac{X_{i}}{O O I P} \times 100
$$

where the original oil in place (OOIP) is the volume of oil initially saturating the micromodel, and $\mathrm{X}_{\mathrm{i}}$ is the volume of oil collected after initial flooding operations. Also additional oil recovery over OOIP (AOR) is the recovered oil in the MEOR operation compared to the amount of oil after water flooding.

\section{RESULTS AND DisCUSSION}

\section{A. Investigation of Grid Independency}

In this section, for attaining optimized number of meshes, grid independency of numerical data from number of cells is checked. For this purpose it has been assumed that water is injected into the micromodel with inlet velocity $10^{-4} \mathrm{~m} / \mathrm{s}$. so three grids with different number of meshes are considered and after water flooding the average velocity of the injected fluid in the porous medium is computed as the objective parameter to investigate the grid independency of results. Table 3 shows conclusion of the grid independency test and the relative error.

As it can be seen in Table III, the relative error between grid 1 and 2 is small and for lowering the time of calculation, grid 1 with $1.1 \times 10^{5}$ is selected as the main grid for MEOR operations.

\begin{tabular}{cccc}
\multicolumn{4}{c}{ TABLE III: RESULTS OF THE GRID INDEPENDENCY } \\
\hline \hline Grid & No. of Cells & $\begin{array}{c}\text { Average velocity } \\
(\mathrm{m} / \mathrm{s})\end{array}$ & $\begin{array}{c}\text { Relative } \\
\text { Error }(\%)\end{array}$ \\
\hline 1 & $1.1 \times 10^{5}$ & $1.12 \times 10^{-4}$ & 2.06 \\
& $2.0 \times 10^{5}$ & $1.15 \times 10^{-4}$ & 2.40 \\
3 & $3.1 \times 10^{5}$ & $1.20 \times 10^{-4}$ & \\
\hline \hline
\end{tabular}

\section{B. Investigating the Accuracy of Simulation Results}

For validating the presented model, simulation results should be compared with the experimental data. So in this research, the simulation results, average $S_{\text {or }}$ and average AOR, are compared with experimental data [16]. Figs. 3 and 4 show the comparison of predicted average $S_{\text {or }}$ and average AOR with experimental data.

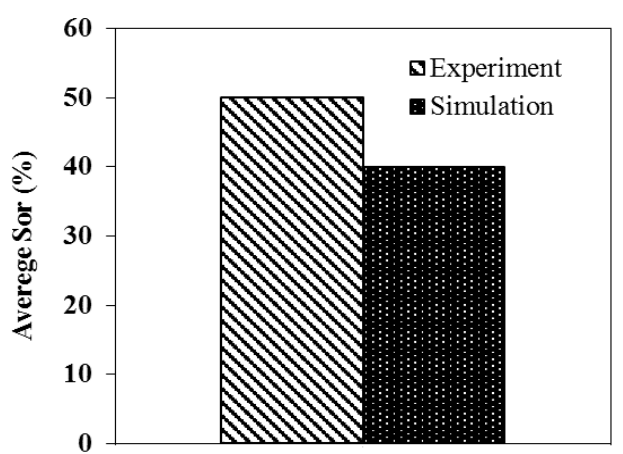

Fig. 3. Comparison of predicted average $S_{\text {or }}$ with experimental data.

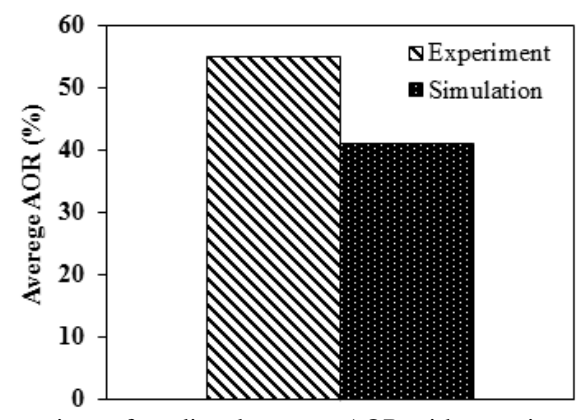

Fig. 4. Comparison of predicted average AOR with experimental data.

(a)

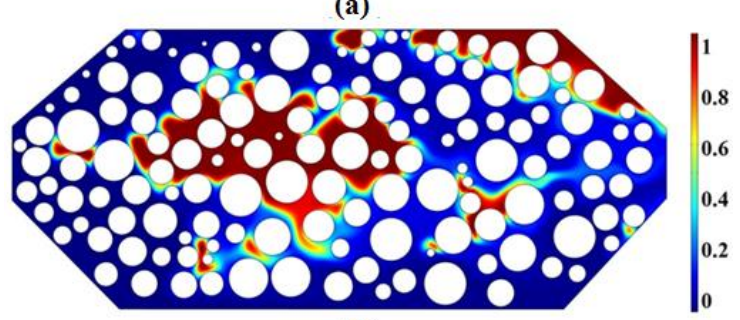

(b)

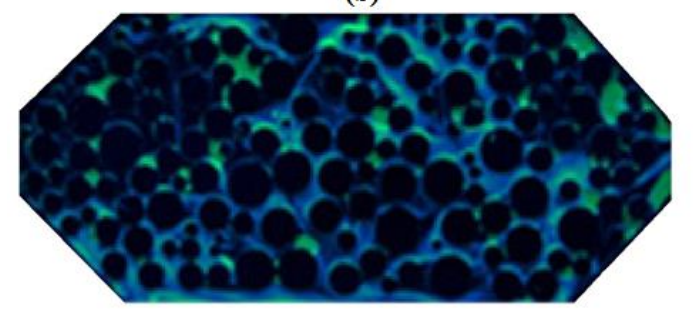

Fig. 5. Visual comparison of residual oil volume fraction in the (a) simulation and (b) experiment.

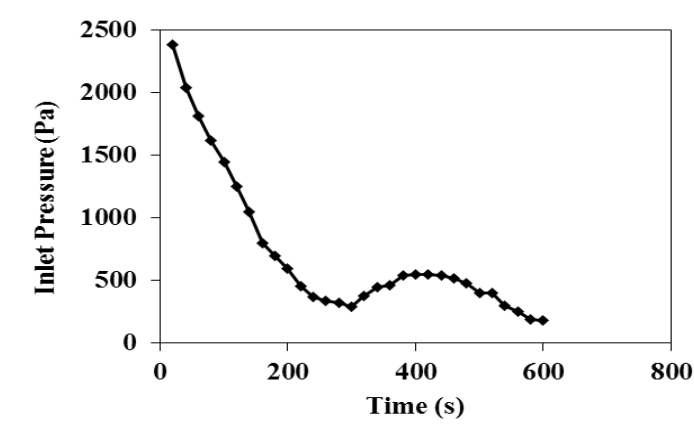

Fig. 6. The inlet pressure of injected fluid versus time.

Also, in Fig. 5 the visual comparison of residual oil volume fraction after MEOR operation in the experiment and simulation test has been presented. In this figure it can be seen that the fluid flow in the simulation run is very similar with flow of the injected fluid in experiment test that it shows the accuracy of simulation model. Of course the observed difference at some points is because of ignoring the effect of bioclogging at the simulated model.

\section{Pressure Profile and Changes of Inlet Pressure}

The pressure profile of the injected fluid has been shown in Fig. 6. As expected, at the entrance of the porous medium the pressure is higher and along the length of the micromodel it decreases till it reaches the minimum value (atmospheric pressure) at the exit port of the porous medium. The inlet pressure of injected fluid versus time has been shown in Fig. 6 . In this figure it can be seen that by injecting the water the rate of decreasing pressure is very high. But by flooding the biosurfactant solution (at $300 \mathrm{sec}$ ) the rate of pressure loss, is 
reduced. It shows that for biosurfactant flooding operations, less equipment is required for pumping the injected fluid.

\section{Oil Displacement}

In Fig. 7, the fluid flow of water at different time has been illustrated. As it can be observed, by starting the injection, the fluid motion path is divided into two branches and continues its movement beside the walls. The reason of this is probably flow channeling near the wall. Also, the fingering effect can be seen clearly into the porous medium. By injecting the biosurfactant solution, the surface tension between injected fluid and oil and the fingering effect consequently decreases. Therefore, the higher amount of oil can be recovered from the porous medium. As shown in Fig. 7, the breakthrough time happens at $240 \mathrm{sec}$.

Fig. 8 shows the volumetric rate of produced oil versus time. The outlet flow rate was calculated by multiplication the velocity of oil at outlet port and outlet cross section. In this figure it can be seen that by biosurfactant flooding at $300 \mathrm{sec}$. the rate of oil production increases that confirms the positive effect of using biosurfactant to increase the recovery factor.

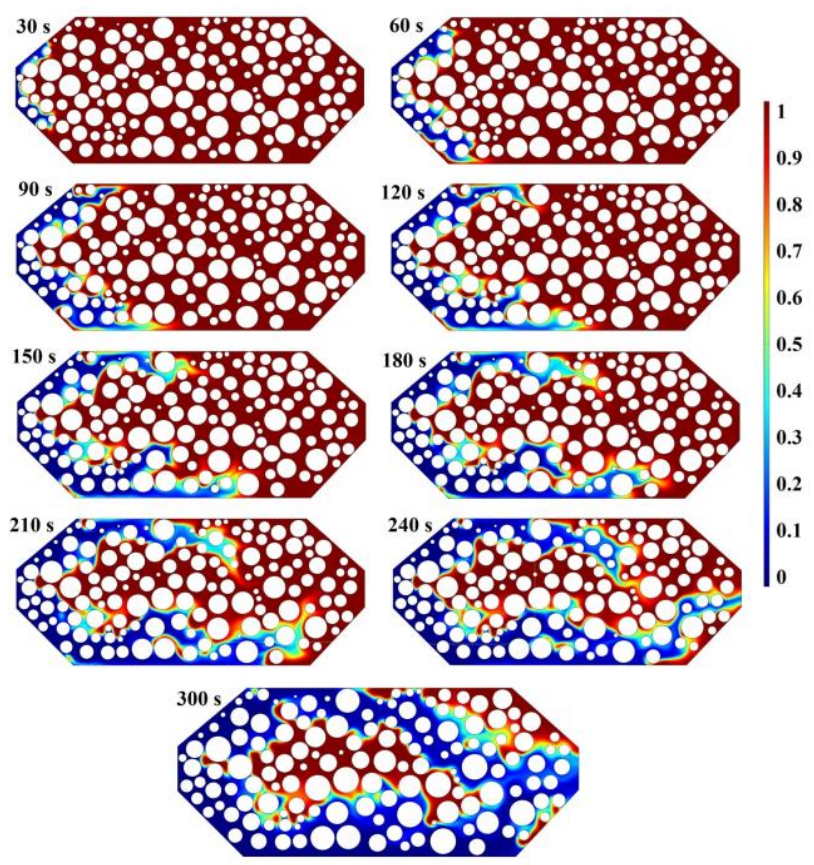

Fig. 7. The oil displacement at different times.

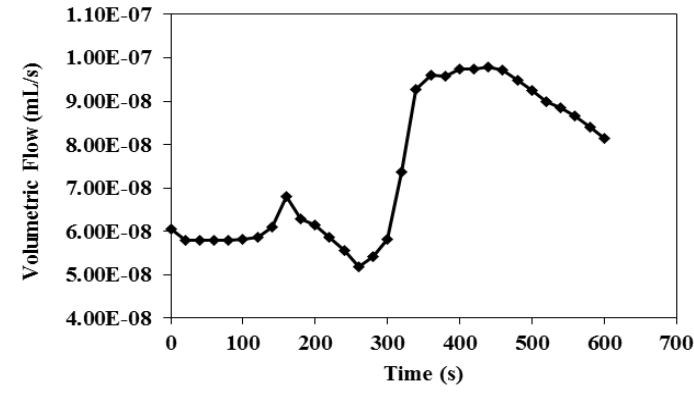

Fig. 8. Volumetric rate of produced oil versus time.

\section{E. Shear Stress Acting on Biosurfactant}

The contour of shear stress in the micromodel has been presented in Fig. 9. Importance of shear stress investigation is because of this fact that the high value of shear stress can damage the biosurfactant. Various biosurfactants have different sensibility toward the shear stress [18]. So lowering the shear stress is very useful to improve the performance of the biosurfactant and increase the oil recovery factor consequently. In Fig. 9 at red regions the damage possibility is more than other zones.

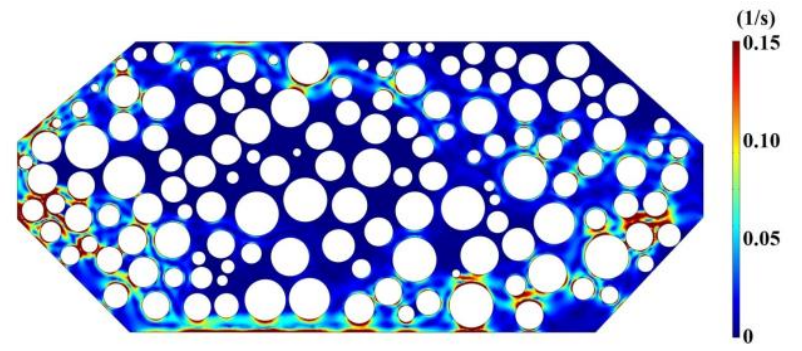

Fig. 9. The contour of shear stress in the micromodel.

\section{F. The Effect of Velocity on the AOR}

Regarding the very low inlet velocity, Reynolds number for the porous medium has been calculated by Eq. 5 [19], which was located in range of laminar flow:

$$
\operatorname{Re}_{p}=\frac{\rho v_{s} d_{p}}{\mu}
$$

The velocity profile of injected fluid into the micromodel has been presented in Fig. 10. Regarding Fig. 7 inlet water flux has been divided into two branches and finally reunited. In Fig. 10 it can be seen that the lower branch has moved faster and as a result has reached to the outlet port faster. Also for have a better visualization some zones are magnified. By investigating the magnified zones in Fig. 10, it is possible to observe movement direction of the injected fluid around pores and pushing the oil through the outlet port. It is worthy to mention that in the port throats of the porous medium, the velocity of injected fluid because of the small space of movement increases.

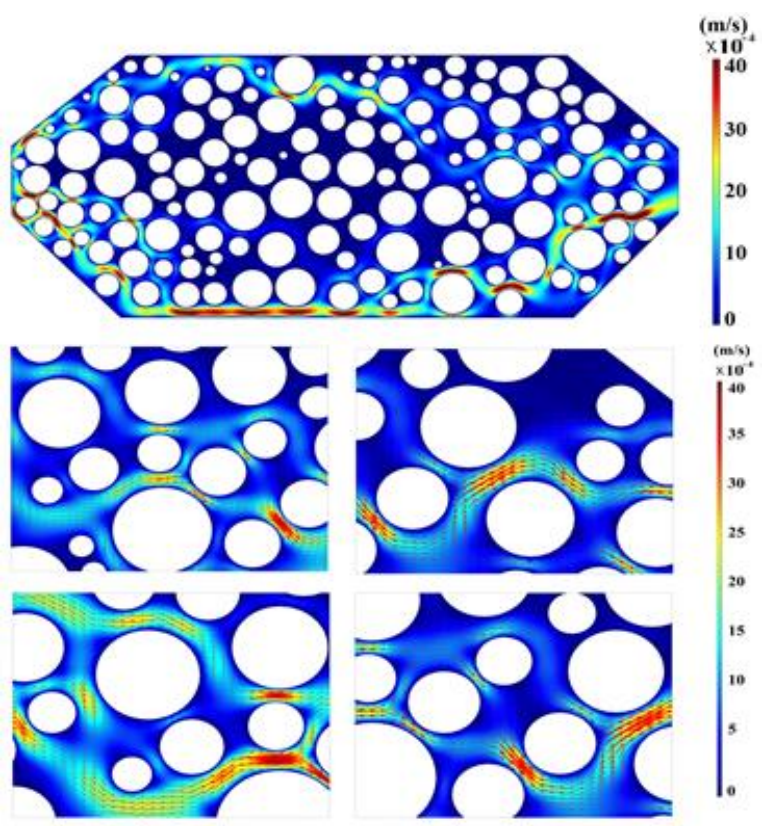

Fig. 10. Velocity profile of the injected fluid into the micromodel.

The effect of inlet velocity on AOR value has been presented in Table IV. By analyzing the results of this table, it 
can be found that by increasing the inlet velocity of the injected fluid, the value of AOR decreases. The reason of this is that by increasing the inlet velocity, water finds its path toward the outlet port quickly and exits the micromodel at lower time, without causing departure of lots of oil [13]. In the other words by increasing the inlet velocity of injected fluid, fingering effect increases, breakthrough time decreases and thus the oil recovery factor reduces.

\begin{tabular}{|c|c|}
\hline Inlet Velocity $(\mathrm{m} / \mathrm{s})$ & AOR (\%) \\
\hline $10^{-2}$ & 38.5 \\
\hline $10^{-3}$ & 39.5 \\
\hline $10^{-4}$ & 41 \\
\hline
\end{tabular}

\section{G. The Effect of Surface Tension on the AOR Value}

The effect of surface tension between two phases (oil and injected fluid) on AOR value has been shown in Fig. 11. In this figure, it can be seen that by decreasing the surface tension, AOR value increases. Because by addition of biosurfactant, the surface tension between the injected fluid and oil decreases. So the fingering effect reduces and the ability of the injected fluid (biosurfactant solution in the second step of the flooding operation) to produce oil from the micromodel increases. Thus by adding biosurfactant to the base fluid and reducing the surface tension, the AOR value improves.

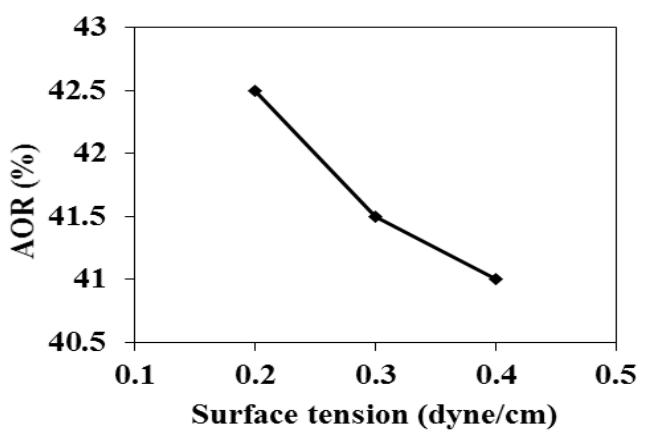

Fig. 11. The effect of surface tension on AOR value.

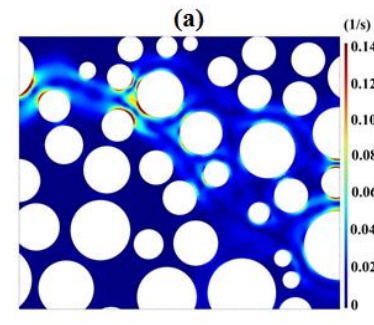

(c)

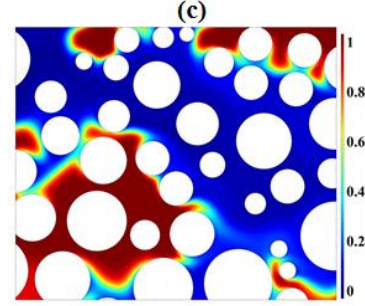

Fig. 12. The profiles of (a) shear stress, (b) pressure, (c) volume fraction of oil and (d) velocity of the injected fluid into the micro model.

Finally to have a better investigation about the effect of biosurfactant solution flooding on the AOR value, a certain zone of the micromodel is magnified and the contour of four parameters including the shear stress, pressure, oil volume fraction and velocity profile of the injected fluid into the micromodel have been presented in Fig. 12.

\section{CONCLUSION}

In this work simulation of biosurfactant flooding into a $2 \mathrm{D}$ micromodel using a computational fluid dynamics technique was performed, and COMSOL Multiphysics 4.3 software was applied to create the geometry and solving the governing equations (momentum, continuity and volume fraction equations) on the system. The numerical results are validated with experimental data and a good agreement was observed between them. The additional oil recovery factor (AOR) is selected as the main objective function to study the effect of biosurfactant flooding in the production of oil from the porous medium. In overall the following results were obtained:

- In the biosurfactant flooding operations, less equipment is required for pumping the injected fluid, because the rate of pressure loss in the biosurfactant flooding is less than water injection.

- By injecting the biosurfactant solution, fingering effect decreases and more surface area of the porous medium will be in contact with the injected fluid. So the oil recovery factor will increase.

- The breakthrough time in the biosurfactant flooding is more than water flooding. Thus MEOR operations have a great potential than water flooding to produce oil from the porous medium.

- Lowering the shear stress on the biosurfactant is very useful to improve its performance in EOR operations.

- By increasing the inlet velocity of the injected fluid, the value of AOR decreases.

- Also, by decreasing the surface tension between oil and injected fluid, AOR value increases and in the biosurfactant flooding operations, the surface tension decreases more than water flooding.

\section{ABBREVIATIONS AND ACRONYMS}

\section{Symbols}

$\begin{array}{ll}d_{p} & \text { Diameter of Circles } \\ \vec{F} & \text { External Body For } \\ \vec{g} & \text { Acceleration of G } \\ i j & \text { Phases } \\ p & \text { Pressure }(\mathrm{Pa}) \\ R e & \text { Reynolds Number } \\ t & \text { Time }(\mathrm{s}) \\ \text { Greek symbols } & \\ \alpha & \text { Volume Fraction } \\ \mu & \text { Viscosity }(\mathrm{Pa} . \mathrm{s}) \\ \vec{v} & \text { Velocity }(\mathrm{m} / \mathrm{s}) \\ \rho & \text { Density }\left(\mathrm{kg} / \mathrm{m}^{3}\right)\end{array}$

\section{REFERENCES}

[1] D. O. Shah, Improved Oil Recovery by Surfactant and polymer Flooding, Elsevier, 2012.

[2] J. Sheng, Modern Chemical Enhanced Oil Recovery: Theory and Practice, Gulf Professional Publishing, 2010. 
[3] H. Rashedi, F. Yazdian, and S. Naghizadeh, Microbial Enhanced Oil Recovery, INTECH Open Access Publisher, 2012.

[4] I. Lazar, I. G. Petrisor, and T. F. Yen, "Microbial enhanced oil recovery (MEOR)," Pet. Sci. Technol., vol. 25, no. 11, pp. 1353-1366, 2007.

[5] J. D. Gaisford, The Economics of Biotechnology, vol. 1, Edward Elgar Publishing, 2001.

[6] M. Gray, A. Yeung, J. Foght, and H. W. Yarranton, "Potential microbial enhanced oil recovery processes: A critical analysis," in Proc. SPE Annual Technical Conference and Exhibition, 2008.

[7] M. Pacwa-Płociniczak, G. A. Płaza, Z. Piotrowska-Seget, and S. S. Cameotra, "Environmental applications of biosurfactants: Recent advances," Int. J. Mol. Sci., vol. 12, no. 1, pp. 633-654, 2011.

[8] S. Thomas, "Enhanced oil recovery-an overview," Oil Gas Sci. Technol. l'IFP, vol. 63, no. 1, pp. 9-19, 2008.

[9] A. Soudmand-asli, S. S. Ayatollahi, H. Mohabatkar, M. Zareie, and S. F. Shariatpanahi, "The in situ microbial enhanced oil recovery in fractured porous media," J. Pet. Sci. Eng., vol. 58, no. 1, pp. 161-172, 2007.

[10] J. F. B. Pereira, E. J. Gudiña, R. Costa, R. Vitorino, J. A. Teixeira, J. A P. Coutinho, and L. R. Rodrigues, "Optimization and characterization of biosurfactant production by Bacillus subtilis isolates towards microbial enhanced oil recovery applications," Fuel, vol. 111, pp. 259-268, 2013.

[11] S. Maudgalya, R. M. Knapp, and M. McInerney, "Microbially enhanced oil recovery technologies: a review of the past, present and future," in Proc. Production and Operations Symposium, 2007.

[12] T. J. Chung, Computational Fluid Dynamics, Cambridge University Press, 2010.

[13] R. Gharibshahi, A. Jafari, A. Haghtalab, and M. S. Karambeigi, "Application of CFD to evaluate pore morphology effect in the nanofluid flooding for enhanced oil recovery," RSC Adv., vol. 5, no. 37 , pp. 28938-28949, 2015.

[14] M. G. Dezfully, A. Jafari, and R. Gharibshahi, "CFD Simulation of Enhanced Oil Recovery using Nanosilica/Supercritical $\mathrm{CO}_{2}, "$ Adv. Mater. Res., vol. 1104, 2015.

[15] R. Gharibshahi, "Simulation and investigation of effective parameters on enhanced oil recovery process using nanoparticles," Tarbiat Modares University, 2014.

[16] R. T. Armstrong and D. Wildenschild, "Investigating the pore-scale mechanisms of microbial enhanced oil recovery," J. Pet. Sci. Eng., vol. 94, pp. 155-164, 2012.

[17] B. A. M. Carciofi, M. Prat, and J. B. Laurindo, "Homogeneous volume-of-fluid (VOF) model for simulating the imbibition in porous media saturated by gas," Energy \& Fuels, vol. 25, no. 5, pp $2267-2273,2011$

[18] A. Pandey, C. R. Soccol, J. A. Rodriguez-Leon, and P. Singh-Nee Nigam, "Solid state fermentation in biotechnology: Fundamentals and applications" Reference Book, Asiatech Publishers, Inc., 2001.

[19] J. Bear, Dynamics of Fuids in Porous Media, Courier Corporation, 2013

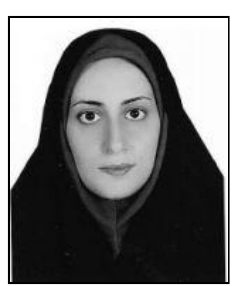

Arezou Jafari was born in 1980. Now, she is an assistance professor at Tarbiat Modares university. She earned his $\mathrm{PhD}$ degree in chemical engineering from Lappeenranta university of technology, Finland.

She has more than 60 papers in ISI journals and international conferences.

Dr. Arezou Jafari is interested in modeling and simulation oil reservoirs, computational fluid dynamics, enhanced oil recovery process, nanofluids, natural gas storage and upgrading of oil products.

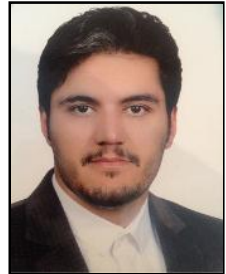

Seyed Ehsan Feghhipour was born on 4 June 1988. $\mathrm{He}$ is an M.S.c graduate student in chemical engineering from Tarbiat Modares University in Tehran, Iran.

He spent his traineeship course in a NGL refinery 1200 in is located near Gachsaran. He has two paper in international conferences and also he has one paper in ISI journals

Mr. Seyed Ehsan Feghhipour is interested in environment, enhanced oil recovery process, nanotechnology and biotechnology.

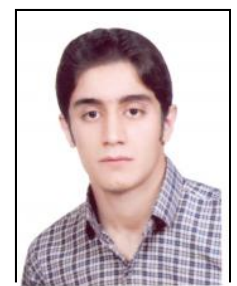

Reza Gharibshahi was born on 21 September 1990 $\mathrm{He}$ is an M.S.c graduate student in chemical engineering from Tarbiat Modares University in Tehran, Iran. He earned his BSC degree in chemical engineering from Petroleum University of Technology, Ahvaz, Iran

$\mathrm{He}$ is a patent analyzer in the Idea Eng. Co. now in Tehran, Iran. He has six papers in international conferences and also he has two papers in ISI journals His research interest lies in the area of computational fluid dynamics, enhanced oil recovery process and nanotechnology. 